\title{
DKK 2016 - diskutieren Sie mit!
}

\author{
Kongresspräsidentin Prof. Dr. Angelika Eggert sowie die Vorsitzenden \\ der Arbeitsgemeinschaft Supportive Maßnahmen in der Onkologie, \\ Rehabilitation und Sozialmedizin (ASORS) der DKG, Prof. Dr. Petra Fey- \\ er und Prof. Dr. Oliver Rick, zum Deutschen Krebskongress 2016.
}

? Frau Professor Eggert, warum wird sich ein Besuch des DKK 2016 lohnen?

Prof. Angelika Eggert: Das Programm des Kongresses weist eine große Themenvielfalt auf und bietet über 300 Sitzungen aus allen Bereichen der Onkologie. Die unterschiedlichen Sichtweisen der über 10.000 erwarteten Teilnehmer und der interdisziplinäre Diskurs können dem eigenen Denken und Handeln enorme Impulse geben, mit denen man in den beruflichen Alltag zurückkehrt.

? Das Kongressmotto des DKK 2016 ist eng an den Begriff der P4-Medizin angelehnt, den der Biomediziner Leroy Hood prägte. Wieso haben Sie sich für dieses Motto entschieden?

Eggert: Die 4 Ps „präventiv, personalisiert, präzise und partizipativ" aus unserem Motto stehen für verschiedene Paradig- menwechsel in der Onkologie, die sich derzeit anbahnen. Wenn wir über Onkologie sprechen, dann liegt das Augenmerk oft auf der modernen Präzisionsmedizin und den neuen Behandlungsstrategien, u.a. aus der personalisierten Medizin. Wir müssen aber auch die Krebsprävention im Blick haben und darüber nachdenken, welche Informationen die Patienten brauchen, um an der Entscheidung über eine Therapie teilhaben zu können und mit ihrer Erkrankung zurechtzukommen.

? Frau Professor Feyer, als eine der beiden ASORS-Vorsitzenden haben Sie aktiv an der Programmplanung zum DKK 2016 mitgewirkt. Welche Themen warten auf die Teilnehmer in diesen Bereichen? Prof. Petra Feyer: Zu den Höhepunkten zählt die Plenarsitzung am ersten Kongresstag, die wir gemeinsam mit der Arbeitsgemeinschaft für Palliativmedizin und der AG Psychoonkologie konzipiert haben. Dort geht es vor allem um die frühe Integration von Palliativmedizin und Supportive Care. Weitere Themen der Plenarsitzung sind die unterstützenden Maßnahmen bei einer ganzen Reihe von Medikamententoxizitäten, auch bei solchen, die im Zusammenhang mit neuen, personalisierten Strategien und Immuntherapien auftreten.

Mit Spannung erwarten wir weiterhin die interdisziplinäre Querschnittsleitlinie „Supportive Therapien bei onkologischen Pati-
entInnen“, die derzeit erarbeitet und auf dem DKK aus Prozesssicht und gegebenenfalls mit den ersten Ergebnissen vorgestellt wird.

? Herr Professor Rick, Sie teilen sich den Vorsitz der ASORS mit Frau Prof. Feyer. Welche Sitzungen werden zum Thema Rehabilitation und Sozialmedizin angeboten?

Prof. Oliver Rick: Ein Teil der sogenannten Langzeitüberlebenden leidet stärker als bisher angenommen unter den Folgen einer Krebsbehandlung, beispielsweise durch Spätschäden, nicht-medizinische Probleme oder gar durch therapiebedingte Zweittumoren. Der Bedarf nach Forschung in diesem Feld ist groß, deshalb wird es eine weitere Plenarsitzung geben, in der es um die lebenslange Begleitung von Krebspatienten geht - von der Rehabilitation als Start für eine erfolgreiche Rückkehr in den Alltag über die Spätfolgen der Behandlung bis hin zur Rückkehr ins Arbeitsleben. Sie sehen, der DKK 2016 bietet ein breites Themenspektrum. Ich kann die Kolleginnen und Kollegen nur ermuntern, den Kongress zu nutzen, um Informationen auszutauschen und mit anderen Experten ins Gespräch zu kommen.

? Und was erhoffen Sie sich, Prof. Eggert, vom DKK 2016?

Eggert: Die Erarbeitung des Kongressprogramms hat dank der Arbeitsgemeinschaften der Deutschen Krebsgesellschaft, der Landeskrebsgesellschaften und der Fachgesellschaften großen Spaß gemacht. Ich freue mich, wenn das Programm des DKK 2016 Anstöße zu einem intensiven, konstruktiven Austausch unter den Teilnehmern gibt. 